\title{
Stereoisomer-Independent Stable Blue Emission in Axial Chiral Difluorenol
}

\author{
Mengna Yu ${ }^{1+}$, Xiong Jia ${ }^{1+}$, Dongqing Lin ${ }^{1}$, Xue Du ${ }^{1}$, Dong Jin ${ }^{1}$, Ying Wei ${ }^{1 *}$, Linghai Xie ${ }^{1,2 *}$ and \\ Wei Huang ${ }^{1,2 *}$
}

${ }^{1}$ Center for Molecular Systems and Organic Devices (CMSOD), State Key Laboratory for Organic Electronics and Information Displays, Institute of Advanced Materials (IAM), Nanjing University of Posts and Telecommunications, Nanjing, China, ${ }^{2}$ Frontiers Science Center for Flexible Electronics (FSCFE), MIIT Key Laboratory of Flexible Electronics (KLoFE), Northwestern Polytechnical University (NPU), Xi'an, China

\section{OPEN ACCESS}

Edited by:

Mengmeng $\mathrm{Li}$,

Institute of Microelectronics (CAS),

China

Reviewed by:

Arkaprabha Konar

Kent State University, United States

Marcelo Henrique Gehlen,

University of São Paulo, Brazil

${ }^{*}$ Correspondence: Ying Wei

iamywei@njupt.edu.cn Linghai Xie

jamlhxie@njupt.edu.cn

Wei Huang

wei-huang@njtech.edu.cn

${ }^{+}$These authors have contributed equally to this work

Specialty section:

This article was submitted to Physical Chemistry and Chemical

Physics,

a section of the journal

Frontiers in Chemistry

Received: 31 May 2021

Accepted: 20 July 2021

Published: 03 September 2021

Citation:

Yu M, Jia X, Lin D, Du X, Jin D, Wei Y,

$X$ ie $L$ and Huang $W$ (2021)

Stereoisomer-Independent Stable

Blue Emission in Axial

Chiral Difluorenol.

Front. Chem. 9:717892.

doi: 10.3389/fchem.2021.717892
Bulky conjugated molecules with high stability are the prerequisite for the overall improvement of performance in wide-bandgap semiconductors. Herein, a chiral difluorenol, 2,2' -(9,9'-spirobi[fluorene]-2,2'-diyl)bis(9-(4-(octyloxy)phenyl)-9H-fluoren-9ol) (DOHSBF), is set as a desirable model to reveal the stereoisomeric effects of widebandgap molecules toward controlling photophysical behavior and improving thermal and optical stability. Three diastereomers are obtained and elucidated by NMR spectra. Interestingly, the effect of modifying the stereo-centers is not observed on optical properties in solutions, pristine films, or post-treated film states. All three diastereomers as well as the mixture exhibit excellent spectral stability without undesirable green emission. Therefore, this stereoisomer-independent blue-emitting difluorenol will be a promising candidate for next-generation wide-bandgap semiconductors that would have extensive application in organic photonics.

Keywords: axial chiral, deep blue, stereoisomer, difluorenol, spirobifluorene

\section{INTRODUCTION}

Organic wide-bandgap blue-emitting semiconductors have attracted more attentions in industrial and fundamental research in information display and solid lighting (Friend et al., 1999; Xie et al., 2012). However, there is one key tough obstacle needed to be overcome before achieving comparable performance with the inorganic counterparts and that is stability (Honmou et al., 2014; Heeger, 2010; Spano and Silva, 2014). The poor color purity and low spectral stability are usually derived from aggregation-induced excimer emission (Farinola and Ragni, 2011; Knaapila and Monkman, 2013), ketone formation (Bliznyuk et al., 1999; Sims et al., 2004), distorted conformation, or entanglement chains (Liu et al., 2016). In addition to device performance, improving the stability of blue luminescent molecules has been a long-standing challenge for plastic electronics. Molecular bulks are favorable for the thermal and morphological stability in organic wide-bandgap semiconductors with potential applications in both information and energy electronics. Bulky groups are the $\mathrm{sp}^{3}$ carbon-containing groups which possess the steric hindrance effect, and functionalized bulky groups were introduced into optoelectronic materials which acted as the suppression of intermolecular force, resulting in the morphological stability (Li et al., 2018; Li et al., 2016; Yu et al., 2019). Therefore, designing bulky conjugated molecules is the prime requirement for light-emitting applications. In the past, the spirobifluorene structure was introduced as the bulk unit to effectively enhance structural rigidity, provide better photothermal stability, and avoid 
fluorescence quenching (Nakagawa et al., 2012; Li et al., 2016). Fluorene-based derivatives were positioned as an important class of blue-emitting semiconductors for their pure blue emission, high photoluminescence quantum efficiency (PLQE), and easy modification (Xie et al., 2012; Yu et al., 2019; Zhu et al., 2019). As a functional position of fluorene units, the chiral $\mathrm{sp} 3$ carbon at the ninth position of the fluorene monomer is an active site for preparing variable stereoisomers and tuning the optoelectronic structure (Karimov and Hartwig, 2018; Pitre et al., 2019). Previously, we demonstrated a supramolecular chiral oligofluorenol, $\quad 2,2^{\prime}$-(spiro[fluorene-9, $9^{\prime}$-xanthene]-2,7-diyl) bis(9-(4-(octyloxy)phenyl)-9H-fluoren-9-ol) (2O8-DPFOHSFX), to investigate the stereoisomerism-property relationship of conjugated aromatic molecules and explore optoelectronic properties (Yuan et al., 2018; Yu et al., 2019). Therefore, precisely controlling the stereochemistry of organic molecules is an important and effective approach for achieving unique photophysical properties.

As chiral structures play a crucial role in sustaining lives, asymmetric synthesis of chiral structures has attracted considerable attention from organic synthetic chemists in many research groups. Early studies mainly focused on central chirality (central atoms with different substituents). Different from molecules with central chirality (point chirality), axial chirality resulting from the steric hindrance of the rotation of the bonds (Nguyen, 2018), has attracted tremendous attention and intensive efforts. The history of the axial chiral structure could date back to 1920 s, when scientists discovered special isomerism of the biphenyl structure (Christie and Kenner, 1922). By 1969, Prelog et al. prepared the first enantiomeric pure spirobifluorene and inspired plenty of scientists to investigate chiral axial compounds, which become a shaft in the research of chiral spiro compounds (Haas and Prelog, 1969). To date, axial chiral molecules not only have been widely used in organic reactions, such as kinetic resolution, asymmetric catalysis, cyclamation/addition, direct aromatization, and chiral recognition, but also exhibit promising application in optoelectronic fields like molecular electronic devices, semiconductors, light-emitting devices, and solar cells (Han et al., 2017; Mishra et al., 2017; Wang and Tan, 2018; Liu Z. S. et al., 2020). Inspired by the supramolecular steric hindrance (SSH) effect (Li et al., 2018), herein, we try to propose a novel strategy based on the molecular integration of steric bulk groups and axial chiral synthons into one functional molecule. We present difluorenol (DOHSBF), which consist of an axial chiral spirobifluorene and two chiral sp3 carbon atoms bearing a phenyl ring and a hydroxyl moiety. Unexpectedly, DOHSBF shows three different stereoisomers, which display stereoisomer-independent stable blue emission.

\section{RESULT AND DISCUSSION}

\section{Material Synthesis and Characterization}

The DOHSBF unit (without alkyl chains) consists of two tertiary alcohols and one spirobifluorene, which form the composition of $\mathrm{C}_{79} \mathrm{H}_{72} \mathrm{O}_{4}$. Theoretically, DOHSBF has six stable stereoisomers due to the three chiral sp3 carbon atoms and the orientation of benzene rings in the molecule, which are $\mathrm{DOHSBF}_{1}$ (aRSS, $C_{2}$ symmetry), $\mathrm{DOHSBF}_{2}$ (aSSS, $C_{2}$ symmetry), $\mathrm{DOHSBF}_{3}$ (aRRS, Asymmetry), $\mathrm{DOHSBF}_{4}$ (aSRS, Asymmetry), $\mathrm{DOHSBF}_{5}(a R R R$, $C_{2}$ symmetry), and $\mathrm{DOHSBF}_{6}$ (aSRR, $C_{2}$ symmetry) (Figure 1A and Supplementary Figure S1), making it an excellent candidate to investigate the stereoisomeric effect of the fluorenol system. These six isomers can be distinguished by different chirality of spirobifluorene and the orientation of benzenes. They can be divided into two pairs of racemes and two mesomers. Among these six stable stereoisomers obtained from the structural optimization via quantum calculation, $\mathrm{DOHSBF}_{1}, \mathrm{DOHSBF}_{2}$, $\mathrm{DOHSBF}_{5}$, and $\mathrm{DOHSBF}_{6}$ seem to show centrosymmetric backbones $\left(C_{2}\right.$ symmetry), which is beneficial to define the actual structures among the isomers. In addition, the optimization results of DOHSBF (Figure 1B) show that the energies of $\mathrm{DOHSBF}_{1}(a R S S)$ and $\mathrm{DOHSBF}_{6}(a S R R)$ structures are the lowest among the six diastereomers. Taking the energy of the aRSS structure $(0 \mathrm{kcal} / \mathrm{moL})$ as a reference, the energy of the other four diastereomers is $0.40 \mathrm{kcal} / \mathrm{moL}\left(\mathrm{DOHSBF}_{2}, a S S S\right)$, $0.48 \mathrm{kcal} / \mathrm{moL}\left(\mathrm{DOHSBF}_{3}, a R R S\right), 0.44 \mathrm{kcal} / \mathrm{moL}\left(\mathrm{DOHSBF}_{4}\right.$, $a S R S)$, and $0.48 \mathrm{kcal} / \mathrm{moL}\left(\mathrm{DOHSBF}_{5}, a R R R\right)$.

Three pairs of DOHSBF diastereoisomers were synthesized via the Suzuki-Miyaura coupling reaction from 2,2'-spirobifluorene (with mixed aS- and aR-axis-chirality) and can be isolated via thin-layer chromatography (Figure 2A). The isomeric features are examined through matrix-assisted laser desorption/ionization time of flight mass spectroscopy (MALDI-ToF-MS). In Figure 2B and Supplementary Figure S2, the mixed DOHSBF samples only exhibit the molecular weight of $1,084.68 \mathrm{~m} / \mathrm{z}$, which is almost identical to isomers 1,2 , and 3 with the molecular weight of 1,084.68, 1,084.55, and 1,084.78, respectively. These results are consistent with the mass simulation of the molecular formula $\mathrm{C}_{79} \mathrm{H}_{72} \mathrm{O}_{4} \quad(1,084.56)$. Furthermore, these isomers properties are reconfirmed by the same number of hydrogen atoms at the aromatic groups, according to the ${ }^{1} \mathrm{H}$ NMR spectra (Figure 2C and Supplementary Figures S3-6) that exhibits the approximately equivalent integration at $7.95-7.85 \mathrm{ppm}(4 \mathrm{H}$, assigning to the $\mathrm{S} 4$ and the S5-position at SBF moiety), $7.70-7.55 \mathrm{ppm}(6 \mathrm{H})$, $7.50-7.20 \mathrm{ppm}(16 \mathrm{H}), 7.15-7.05 \mathrm{ppm}(2 \mathrm{H}$, at the S2-position), $7.05-6.95 \mathrm{ppm}(2 \mathrm{H}$, at the S8-position), and $6.85-6.70 \mathrm{ppm}$ (at the a-site on fluorenol and the S1-position on the SBF group). Even so, there are some subtle differences in chemical shift of hydrogen signals, probably derived from the integrated tactic effects of SBF-based axis chirality (Hamada et al., 2020) and asymmetric fluorenol chirality (Yuan et al., 2018; Yu et al., 2019; Wei et al., 2019). At $7.00 \mathrm{ppm}$, isomers 1 and 3 possess the singlet peak of S8 at 6.98 and $6.96 \mathrm{ppm}$, respectively, while isomer 2 exhibits the splitting multiple S8 peaks in this region. The splitting signals are also observed in 3.95-3.80 ppm (assigning to alkoxyl chains pendant on fluorenol groups) and 7.15-7.05 ppm for isomer 2, which suggests that the asymmetric backbones is consistent with the meso-DOHSBF type ( $\mathrm{DOHSBF}_{3}$ and $\mathrm{DOHSBF}_{4}$, in Figure 1A). The symmetric structures without splitting in isomer 1 are assigned to rac-DOHSBF types, as is agreement with the simulated $C_{2^{-}}$ symmetric results (Figure 1B). These results are consistent with SFX-based difluorenol building blocks where the meso-configuration 
A
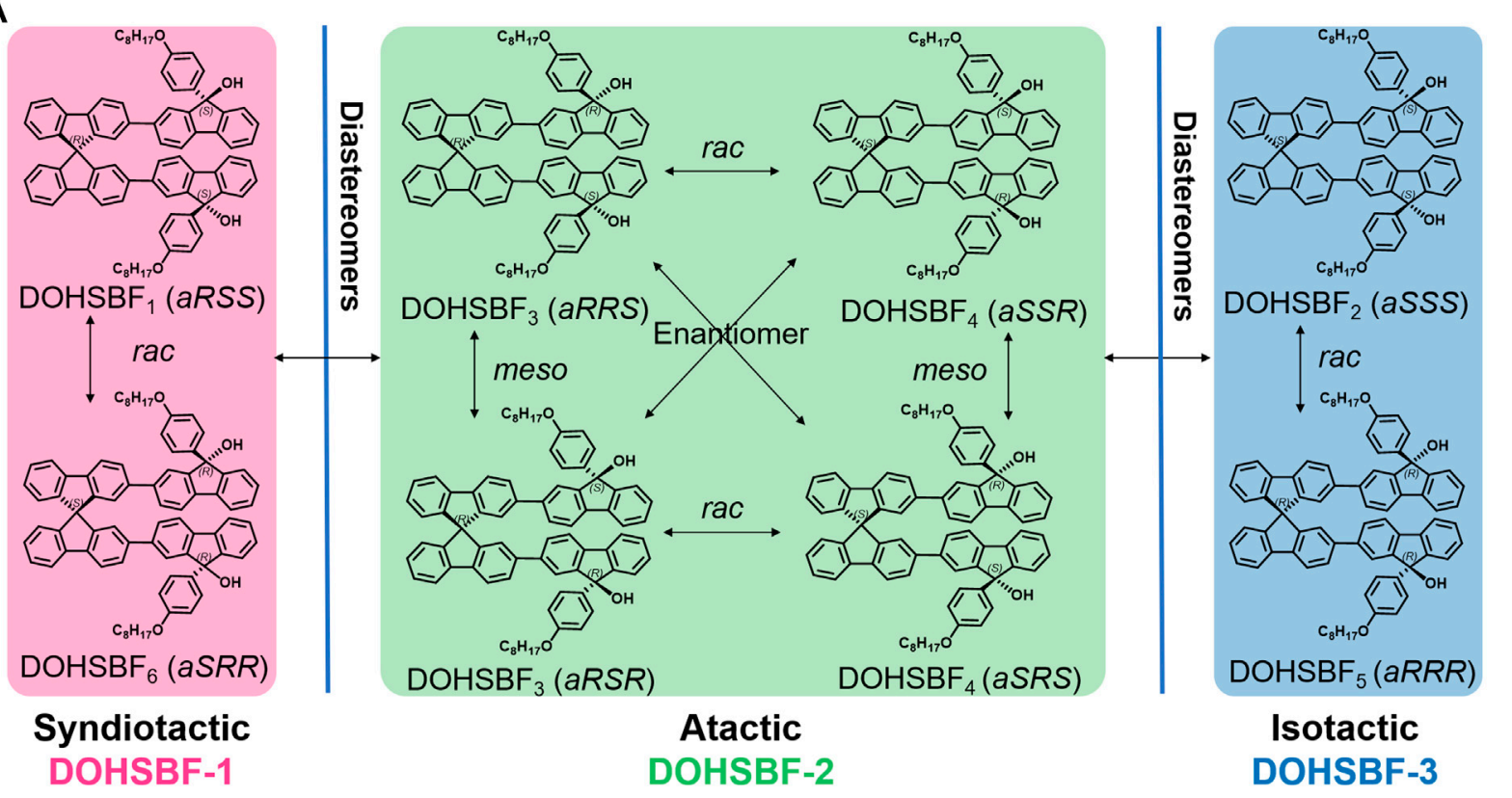

B

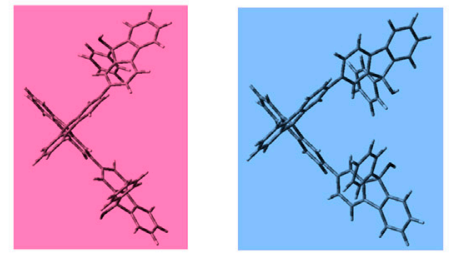

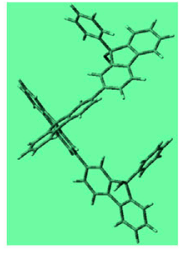

$\mathrm{DOHSBF}_{3}$

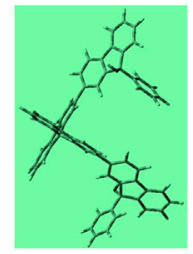

$\mathrm{DOHSBF}_{4}$
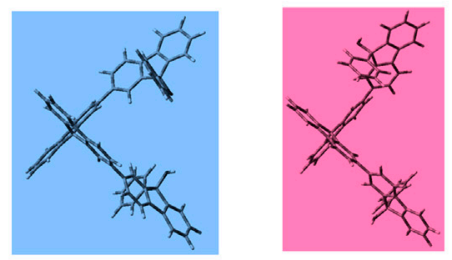

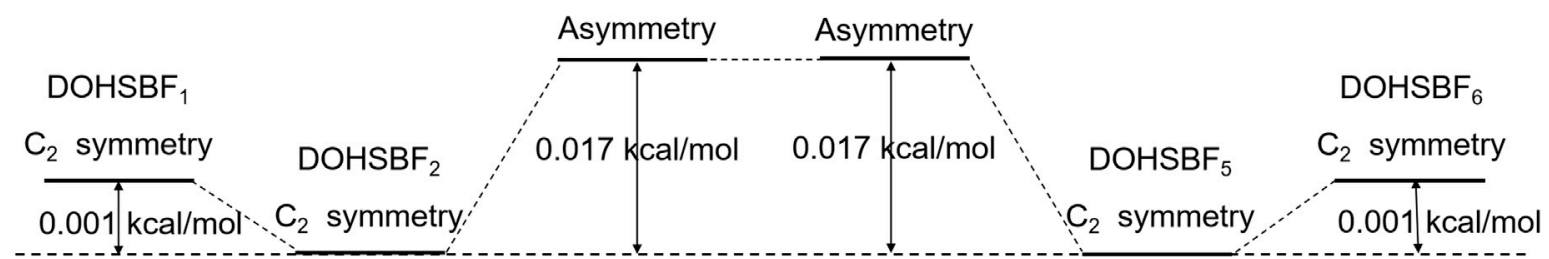

FIGURE 1 | (A) Theoretical configurations of DOHSBF. (B) Energy calculation comparison of possible theoretical stereoisomers of DOHSBF (alkyl chains are omitted) by Gaussian 09 at the B3LYP/6-31G(d) level.

exhibits the asymmetrically splitting feature on proton resonance (Yuan et al., 2018; Yu et al., 2019). Meanwhile, it is suggested that SBF-based axis chirality does not additionally break the symmetry of steric structures, as is in agreement with the tactic effects of other axis-chiral groups such as chiral binol-phosphate esters (Xiang et al., 2020) and 9,9' -spirobifluoren-derivates (Haas and Prelog, 1969).

\section{Thermal and Electrochemical Properties}

As depicted in Figure 3A and Supplementary Figure S7, all three stereoisomers show good solubility in organic solvents, such as dichloromethane, tetrahydrofuran, chloroform, and ethyl acetate, and exhibit a deep blue color. But the solubility of DOHSBF-1 is better than DOHSBF-2 in dichloromethane, and then DOHSBF-3. The X-ray powder diffraction (XRD) patterns in Supplementary Figure S8 show no apparent diffraction peaks, indicating that when pure, all the isomers formed amorphous films. The thermal stability is confirmed using the thermogravimetry analysis (TGA) and differential scanning calorimetry (DSC) measurements. Decomposition temperatures $\left(5 \%\right.$ weight loss, termed $\left.\mathrm{T}_{\mathrm{d}}\right)$ recorded in order are mixture $\left(315^{\circ} \mathrm{C}\right)>$ isomer-3 $\left(278^{\circ} \mathrm{C}\right)>$ isomer-1 $\left(276^{\circ} \mathrm{C}\right)>$ isomer-2 $\left(261^{\circ} \mathrm{C}\right) \quad$ (Figure $3 \mathrm{~B}$ and Supplementary Figure S9). These high $\mathrm{T}_{\mathrm{d}}$ values suggested good thermal stability of DOHSBF for its application in optoelectronic devices, and the mixture of difluorenol possesses the enhanced stability during the evaporation process. The DSC curves indicate that three pure difluorenol derivatives show no glass phase transition and melting point by heating to $178^{\circ} \mathrm{C}$, but the mixture presents an inconspicuous glassy transition temperatures $\left(\mathrm{T}_{\mathrm{g}}\right)$ at $114^{\circ} \mathrm{C}$ (Supplementary Figure S10). The cyclic voltammetry (CV) measurement was carried out to investigate the electrochemical oxidation and reduction behaviors of DOHSBF and estimated the corresponding HOMO and LUMO energy levels. The CV curves of 


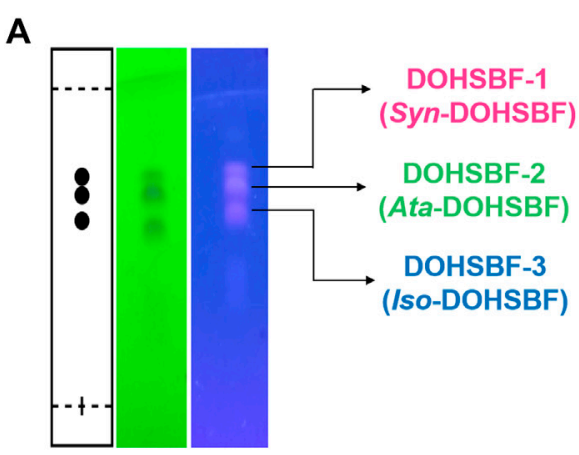

C

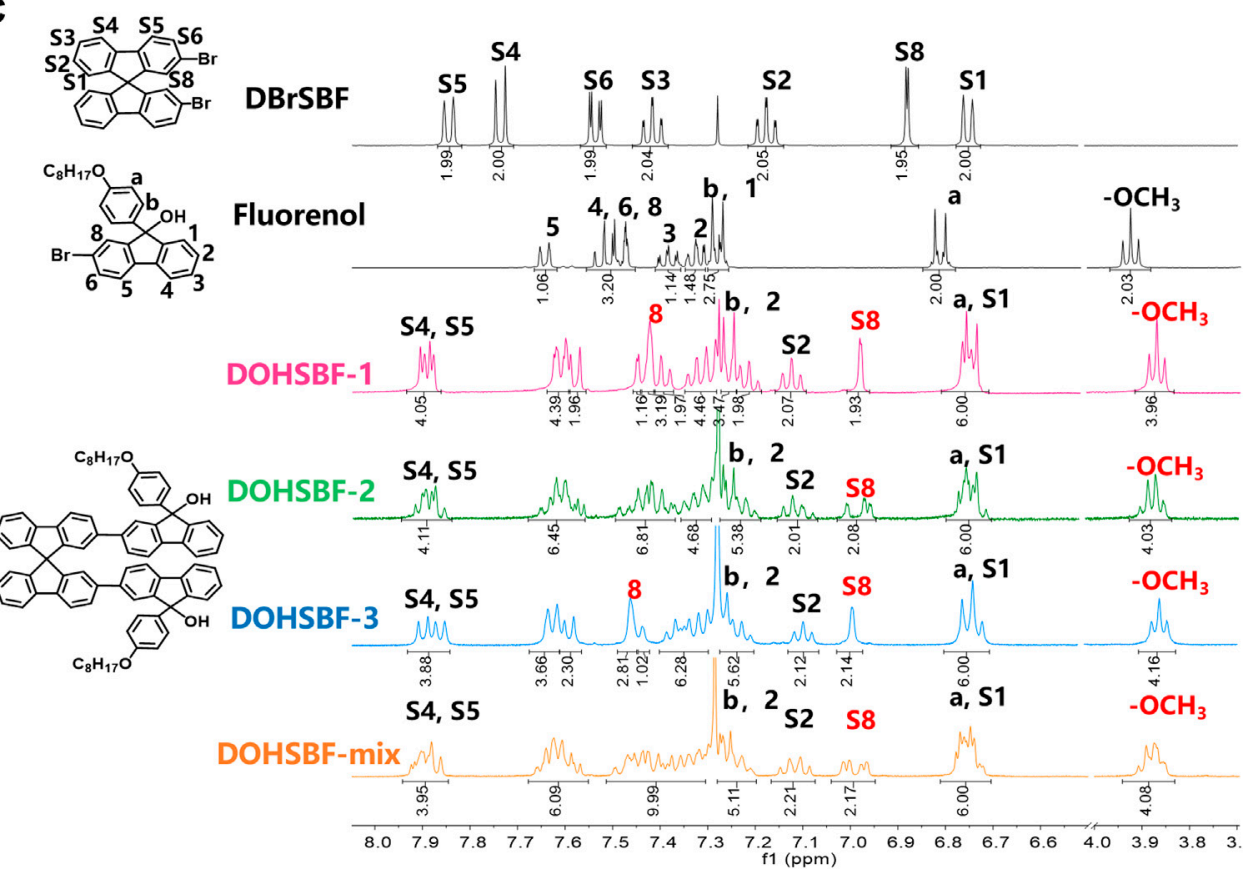

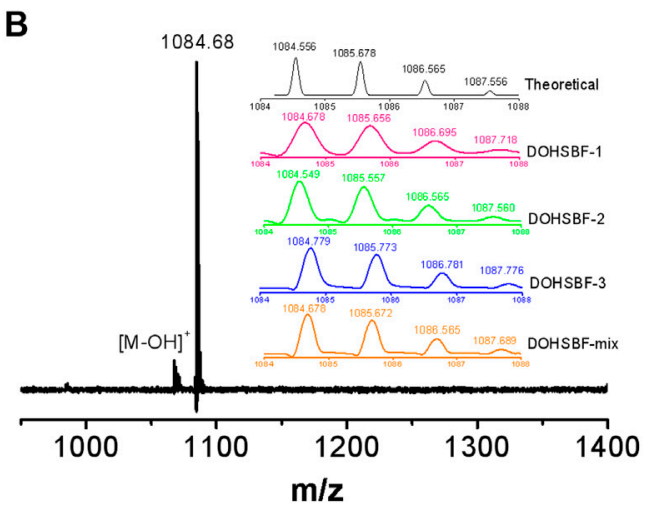

B 

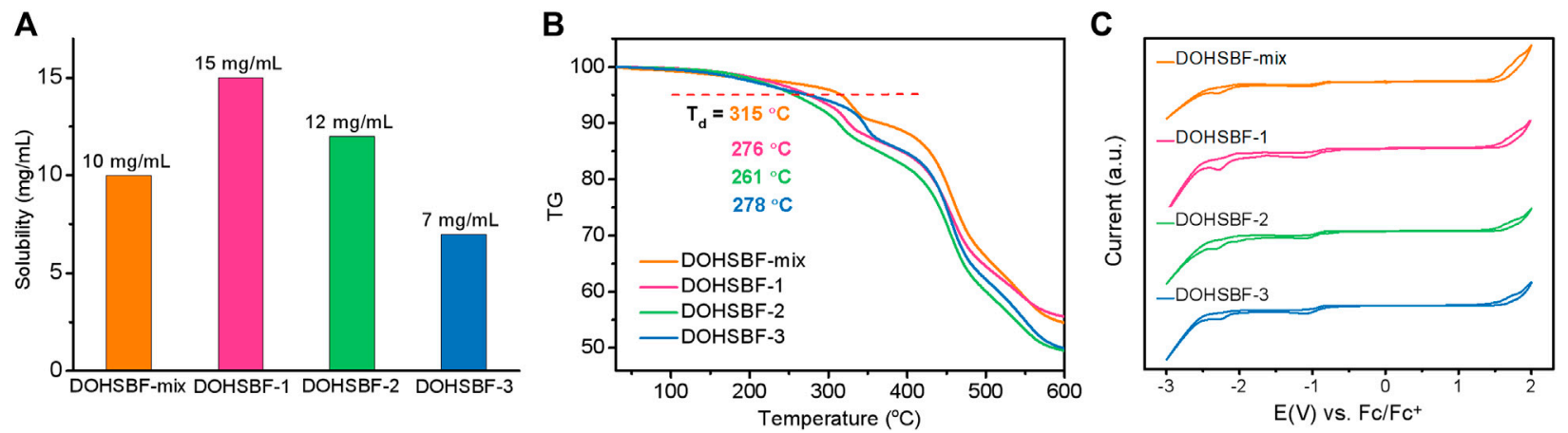

FIGURE 3 | (A) The solubility of DOHSBF in dichloromethane. (B) TGA curves and (C) cyclic voltammogram of DOHSBF.
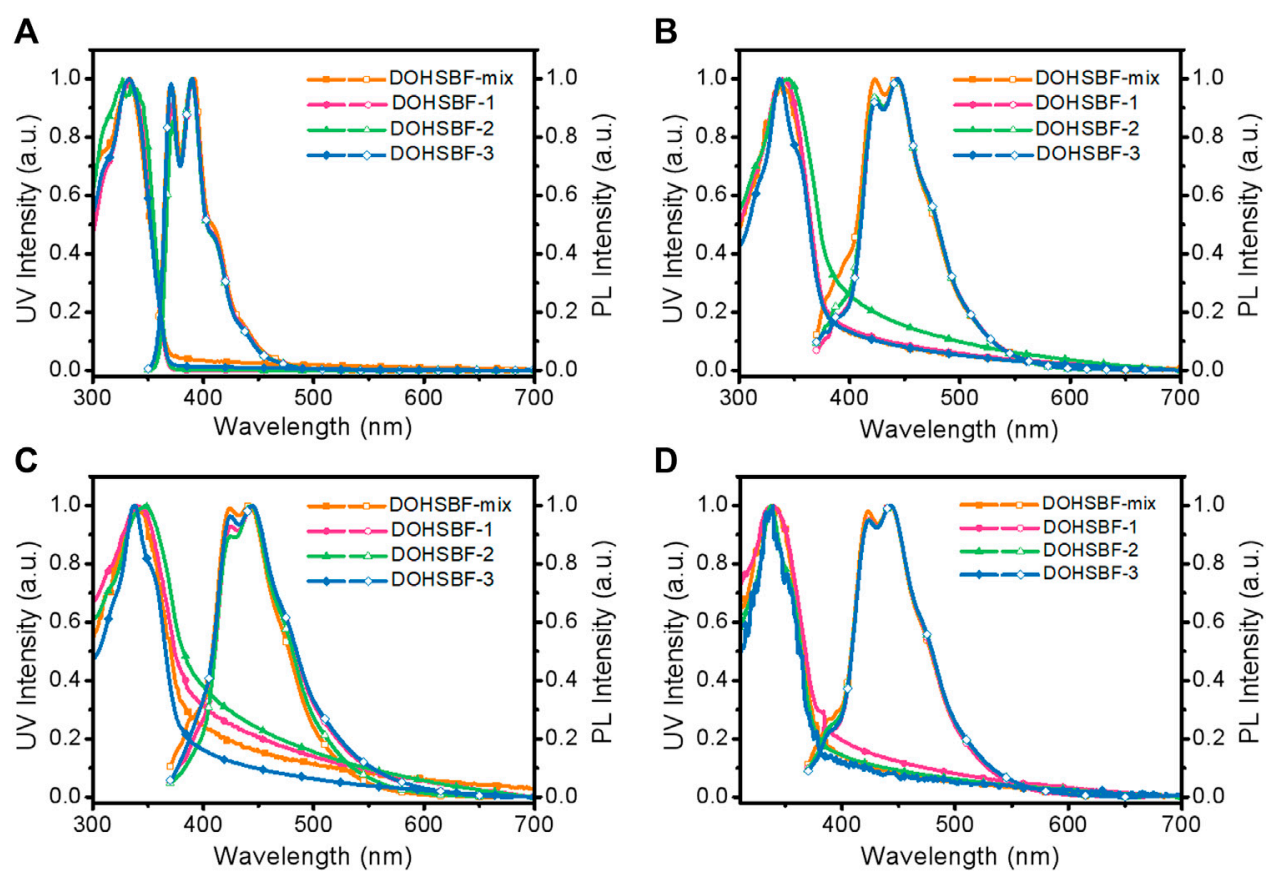

FIGURE 4 | Optical properties of DOHSBF in various states. UV-vis absorption and photoluminescence (PL) spectra of DOHSBF (A) dilute solutions, (B) spincoated pristine films, (C) annealed films at $220^{\circ} \mathrm{C}$ for $10 \mathrm{~min}$, and (D) photooxidation films, respectively.

pristine films are shown in Figure 4B. Similarly, the emission spectra of them in the film state present three emission bands at about 423, 443, and $471 \mathrm{~nm}$, and the corresponding $0-1$ transition in the film are bathochromic to $443 \mathrm{~nm}$, compared to the solution states. In addition, thermal annealing at high temperature in air was further conducted to study the spectral stability for the isomer films. It can be seen from the Figure 4C and the Supplementary Figure S11 that there is almost no change in the UV-vis spectra of DOHSBF films after annealing in air for $10 \mathrm{~min}$. With regard to fluorescence emission spectra, there is no difference after annealing, illustrating that the DOHSBF has relatively good thermal stability. Compared to the conventional thermal stability, the oxidation stability of luminescent films has been rarely addressed in the last several decades, which is the most important factor determining practical optoelectronic application in the future. Herein, we make a further research study on the influence of chiral forms by photooxidation of pristine spin-coated films. Experimental procedure for photooxidation measurement involves exposing the pristine films under an ultraviolet lamp $(365 \mathrm{~nm})$ irradiation for $30 \mathrm{~min}$. As presented in Figure 4D, no obvious change of absorption and emission behaviors was found in all four samples, and the green emission band with the $\mathrm{I}_{\text {green }} /$ $\mathrm{I}_{\text {blue }}$ ratio (the ratio of emission intensities at 550 and $443 \mathrm{~nm}$ ) is very low, indicating its excellent deep blue emission spectral stability without undesirable green emission. Unlike previously reported difluorenol molecule, 9,9'-diphenyl-9H,9'H-[2,2'bifluorene]-9, $9^{\prime}$-diol (DPFOH), it was observed that all PL 
TABLE 1 | Photophysical properties of the isomers at various states.

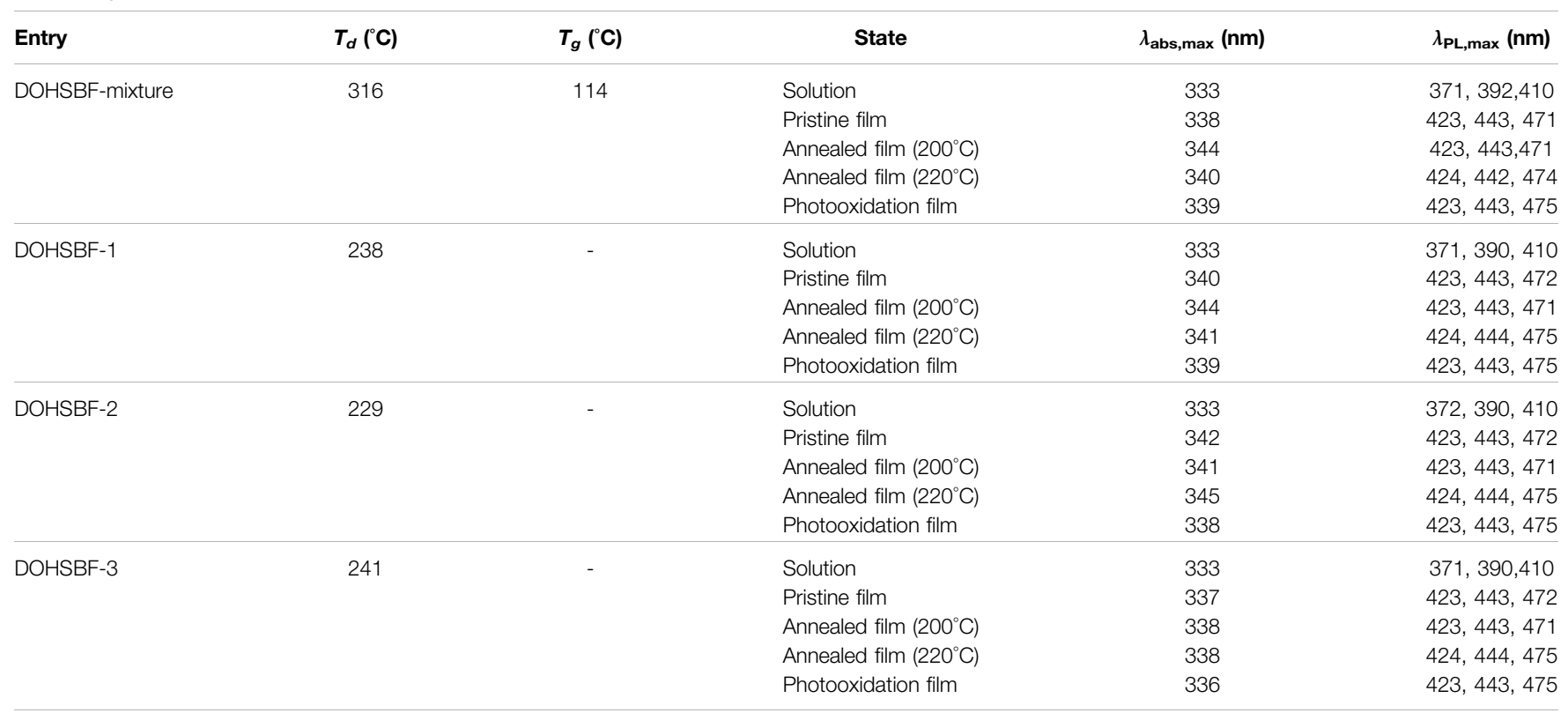

spectra of the isomers, either the solutions or the films, changed conspicuously with the appearance of an additional shoulder of green-band emission under thermal annealing and UV irradiation oxidation (Liu Y. et al., 2020). Compared to DOHSBF, another difluorenol bulky molecule with the similar structure, 2O8-DPFOH-SFX, its raceme and mesomer display the distinct conformations and optoelectronic properties in their condensed states. The annealed meso-2O8-DPFOH-SFX film has a stronger green emission band at $510 \mathrm{~nm}$ with an $\mathrm{I}_{\text {green }} /$ $\mathrm{I}_{\text {blue }}$ ratio of 0.5 , but no obvious change in the green band was observed for the annealed rac-2O8-DPFOH-SFX film $\left(\mathrm{I}_{\text {green }} / \mathrm{I}_{\text {blue }}\right.$ $=0.143$ ), indicating stereoisomerism sensitive PL property ( $\mathrm{Yu}$ et al., 2019). In this regard, only the DOHSBF molecules, either isomers or the mixture, show stereoisomer-insensitive deep blue emission with enhanced stability. Therefore, in spite of the diverse diastereomers, a similar conjugated backbone plays a more key role in dominating optical properties in accordance with the identical absorption and emission spectra of stereoisomers in solution and film states.

For profound understanding of the photophysical properties, then we measured the photoluminescence quantum yield (PLQY) of solutions, pristine films, and annealed films using an integrating sphere. The PLQY values of DOHSBF dilute solutions are relatively high (all four are about $80 \%$ ) owing to the single-molecular excitonic behavior. Accordingly, the fluorescence PLQY of DOHSBF-mix pristine and annealed films were measured to be $\Phi_{\mathrm{f}}=13$ and $18 \%$, respectively, much lower than that of the solution state, owing to the aggregation-caused quenching (ACQ). The PLQYs of pure stereoisomeric analogues were also measured, with $\Phi_{\mathrm{f}}$ of 12 and $14 \%$ for isomer-1 pristine and the annealed film, 13 and $16 \%$ for isomer- 2 pristine and the annealed film, 14 and $20 \%$ for isomer-3 pristine and the annealed film, respectively. Compared to the initial films, the annealed one shows higher $\Phi_{\mathrm{f}}$ values, which may be attributed to molecular geometry optimization and ordering under thermal activation, and thus preventing intermolecular exciton coupling and excimer emission. Accordingly, the annealing enhanced fluorescence quantum yield also contributes to stable fluorescence emission.

\section{CONCLUSION}

In summary, we demonstrated the stereoisomeric effects of DOHSBF on the photophysical behavior. Impressively, the stereoisomeric effect on optical properties in solutions, spincoated films, and post-treated film states is negligible. As compared to the difluorenols reported in previous literature, we found that DOHSBF in each form exhibit excellent blue spectral stability without undesirable green emission. Spatial isomerism in organic molecule of stereoisomers can not only precisely uncover the structure-function relationship but also play a key role in opening up new design strategies for organic functional materials.

\section{DATA AVAILABILITY STATEMENT}

The original contributions presented in the study are included in the article/Supplementary Material; further inquiries can be directed to the corresponding authors.

\section{AUTHOR CONTRIBUTIONS}

MY and XJ: designed experiments. MY, XJ, and XD: carried out experiments. MY, XJ, DL, DJ, YW, LX, and WH: analyzed experimental results. $\mathrm{MY}$ and $\mathrm{XJ}$ : wrote the manuscript. All 
authors contributed to the article and approved the submitted version.

\section{FUNDING}

The study was supported by the National Natural Science Foundation of China (21774061, 22075136, 61874053), "HighLevel Talents in Six Industries" of Jiangsu Province (XYDXX019), the Open Project from State Key Laboratory of Supramolecular Structure and Materials at Jilin University (No. sklssm202108), the China Postdoctoral Science

\section{REFERENCES}

Bliznyuk, V. N., Carter, S. A., Scott, J. C., Klärner, G., Miller, R. D., and Miller, D. C. (1999). Electrical and Photoinduced Degradation of Polyfluorene Based Films and Light-Emitting Devices. Macromolecules 32, 361-369. doi:10.1021/ ma9808979

Christie, G. H., and Kenner, J. (1922). LXXI.-The Molecular Configurations of Polynuclear Aromatic Compounds. Part I. The Resolution of $\gamma-6: 6^{\prime}-$ dinitro- and $4: 6: 4^{\prime}: 6^{\prime}$-Tetranitro-Diphenic Acids into Optically Active Components. J. Chem. Soc. Trans. 121, 614-620. doi:10.1039/ CT9222100614

Farinola, G. M., and Ragni, R. (2011). Electroluminescent Materials for white Organic Light Emitting Diodes. Chem. Soc. Rev. 40, 3467-3482. doi:10.1039/ C0CS00204F

Friend, R. H., Gymer, R. W., Holmes, A. B., Burroughes, J. H., Marks, R. N., Taliani, C., et al. (1999). Electroluminescence in Conjugated Polymers. Nature 397, 121-128. doi:10.1038/16393

Haas, G., and Prelog, V. (1969). Optisch Aktive 9,9'-Spirobifluoren-Derivate. Helv. Chim. Acta 52 (5), 1202-1218. doi:10.1002/hlca.19690520505

Hamada, H., Itabashi, Y., Shang, R., and Nakamura, E. (2020). Axially Chiral SpiroConjugated Carbon-Bridged P-Phenylenevinylene Congeners: Synthetic Design and Materials Properties. J. Am. Chem. Soc. 142, 2059-2067. doi:10.1021/jacs.9b13019

Han, J., Duan, P., Li, X., and Liu, M. (2017). Amplification of Circularly Polarized Luminescence through Triplet-Triplet Annihilation-Based Photon Upconversion. J. Am. Chem. Soc. 139, 9783-9786. doi:10.1021/ jacs.7b04611

Heeger, A. J. (2010). Semiconducting Polymers: the Third Generation. Chem. Soc. Rev. 39, 2354-2371. doi:10.1039/B914956M

Honmou, Y., Hirata, S., Komiyama, H., Hiyoshi, J., Kawauchi, S., Iyoda, T., et al. (2014). Single-Molecule Electroluminescence and Photoluminescence of Polyfluorene Unveils the Photophysics Behind the Green Emission Band. Nat. Commun. 5, 4666. doi:10.1038/ ncomms 5666

Karimov, R. R., and Hartwig, J. F. (2018). Transition-Metal-Catalyzed Selective Functionalization of C(sp3 )-H Bonds in Natural Products. Angew. Chem. Int. Ed. 57, 4234-4241. doi:10.1002/anie.201710330

Knaapila, M., and Monkman, A. P. (2013). Methods for Controlling Structure and Photophysical Properties in Polyfluorene Solutions and Gels. Adv. Mater. 25, 1090-1108. doi:10.1002/adma.201204296

Li, S., Liu, W., Shi, M., Mai, J., Lau, T.-K., Wan, J., et al. (2016). A Spirobifluorene and Diketopyrrolopyrrole Moieties Based Non-fullerene Acceptor for Efficient and Thermally Stable Polymer Solar Cells with High Open-Circuit Voltage. Energy Environ. Sci. 9, 604-610. doi:10.1039/ c5ee03481g

Li, Y. X., Wang, S. S., Yu, Y., Zhang, H., Wang, W. Y., Yang, R. Q., et al. (2018). SMART Design of a Bulk-Capped Supramolecular Segment for the Assembly into Organic Interdigital Lipid Bilayer-Like (ILB) Nanosheets. Small 14, 1703151. doi:10.1002/smll.201703151

Liu, Y.-Y., Lin, J.-Y., Bo, Y.-F., Xie, L.-H., Yi, M.-D., Zhang, X.-W., et al. (2016). Synthesis and Crystal Structure of Highly Strained [4]
Foundation (2020M671459 and 2020M671555), the NUPT Scientific Foundation (NY220086), the Jiangsu Province Postdoctoral Research Fund (SBH20005), and the China National Postdoctoral Program for Innovative Talents (BX20200170).

\section{SUPPLEMENTARY MATERIAL}

The Supplementary Material for this article can be found online at: https://www.frontiersin.org/articles/10.3389/fchem.2021.717892/ full\#supplementary-material

Cyclofluorene: Green-Emitting Fluorophore. Org. Lett. 18, 172-175. doi:10.1021/acs.orglett.5b03038

Liu, Y., Bo, Y., Xie, X., Yan, Y., Xu, M., Ou, C., et al. (2020). DiastereoisomerInduced Morphology Tunable Self-Assembled Organic Microcrystals of Conjugated Molecules for Ultraviolet Laser. Adv. Mater. Inter. 7, 1902057. doi:10.1002/admi.201902057

Liu, Z.-S., Hua, Y., Gao, Q., Ma, Y., Tang, H., Shang, Y., et al. (2020). Construction of Axial Chirality via Palladium/chiral Norbornene Cooperative Catalysis. Nat. Catal. 3, 727-733. doi:10.1038/s41929-0200494-1

Mishra, S., Liu, J., and Aponick, A. (2017). Enantioselective Alkyne Conjugate Addition Enabled by Readily Tuned Atropisomeric P,N-Ligands. J. Am. Chem. Soc. 139, 3352-3355. doi:10.1021/jacs.7b00363

Nakagawa, T., Ku, S.-Y., Wong, K.-T., and Adachi, C. (2012). Electroluminescence Based on Thermally Activated Delayed Fluorescence Generated by a Spirobifluorene Donor-Acceptor Structure. Chem. Commun. 48, 9580-9582. doi:10.1039/c2cc31468a

Nguyen, D. T. (2018). Giving Atropisomers Another Chance. Chem. Eng. News 96, 22-25. doi:10.1021/cen-09633-feature1

Pitre, S. P., Weires, N. A., and Overman, L. E. (2019). Forging C(sp3)-C(sp3) Bonds with Carbon-Centered Radicals in the Synthesis of Complex Molecules. J. Am. Chem. Soc. 141, 2800-2813. doi:10.1021/jacs.8b11790

Sims, M., Bradley, D. D. C., Ariu, M., Koeberg, M., Asimakis, A., Grell, M., et al. (2004). Understanding the Origin of the $535 \mathrm{Nm}$ Emission Band in Oxidized Poly(9,9-Dioctylfluorene): The Essential Role of Inter-Chain/ Inter-Segment Interactions. Adv. Funct. Mater. 14, 765-781. doi:10.1002/ adfm. 200305149

Spano, F. C., and Silva, C. (2014). H- and J-Aggregate Behavior in Polymeric Semiconductors. Annu. Rev. Phys. Chem. 65, 477-500. doi:10.1146/annurevphyschem-040513-103639

Wang, Y.-B., and Tan, B. (2018). Construction of Axially Chiral Compounds via Asymmetric Organocatalysis. Acc. Chem. Res. 51, 534-547. doi:10.1021/ acs.accounts.7b00602

Wei, Y., Luo, M., Zhang, G., Lei, J., Xie, L.-H., and Huang, W. (2019). A Convenient One-Pot Nanosynthesis of a C(sp2)-C(sp3)-Linked 3D Grid via an 'A2 + B3' Approach. Org. Biomol. Chem. 17, 6574-6579. doi:10.1039/C9OB00754G

Xiang, D. F., Narindoshvili, T., and Raushel, F. M. (2020). Atropselective Hydrolysis of Chiral Binol-Phosphate Esters Catalyzed by the Phosphotriesterase from Sphingobium Sp. TCM1. Biochemistry 59, 4463-4469. doi:10.1021/acs.biochem.0c00831

Xie, L.-H., Yin, C.-R., Lai, W.-Y., Fan, Q.-L., and Huang, W. (2012). Polyfluorenebased Semiconductors Combined with Various Periodic Table Elements for Organic Electronics. Prog. Polym. Sci. 37, 1192-1264. doi:10.1016/ j.progpolymsci.2012.02.003

Yu, M.-N., Lin, J.-Y., Li, Y.-X., Soleimaninejad, H., Ou, C.-J., Bai, L.-B., et al. (2019). Emission Enhanced and Stabilized by Stereoisomeric Strategy in Hierarchical Uniform Supramolecular Framework. Chem 5, 2470-2483. doi:10.1016/ j.chempr.2019.06.021

Yuan, X.-A., Yu, M.-N., Zhu, Q., Zhang, W.-W., Xie, L.-H., Huang, W., et al. (2018). Synergistic Steric Pairing Effects of Terfluorenes with Ternary Side Groups on $\beta$-conformation Transition: Experiments and Computations. J. Mater. Chem. C 6, 1551-1561. doi:10.1039/c7tc04817c 
Zhu, W.-S., Han, Y.-M., An, X., Weng, J.-N., Yu, M.-N., Bai, L.-B., et al. (2019). Highly Emissive Hierarchical Uniform Dialkylfluorene-Based Dimer Microcrystals for Ultraviolet Organic Laser. J. Phys. Chem. C 123, 28881-28886. doi:10.1021/acs.jpcc.9b07895

Conflict of Interest: The authors declare that the research was conducted in the absence of any commercial or financial relationships that could be construed as a potential conflict of interest.

Publisher's Note: All claims expressed in this article are solely those of the authors and do not necessarily represent those of their affiliated organizations, or those of the publisher, the editors and the reviewers. Any product that may be evaluated in this article, or claim that may be made by its manufacturer, is not guaranteed or endorsed by the publisher.

Copyright $\odot 2021 \mathrm{Yu}$, Jia, Lin, Du, Jin, Wei, Xie and Huang. This is an open-access article distributed under the terms of the Creative Commons Attribution License (CC $B Y)$. The use, distribution or reproduction in other forums is permitted, provided the original author(s) and the copyright owner(s) are credited and that the original publication in this journal is cited, in accordance with accepted academic practice. No use, distribution or reproduction is permitted which does not comply with these terms. 